\title{
Glucose Induced Permeation Control of Insulin through a Complex Membrane Consisting of Immobilized Glucose Oxidase and a Poly(amine)
}

\author{
Kazuhiko Ishihara, * Mineko Kobayashi, Naoshi IshImarU, \\ and Isao SHINOHARA \\ Department of Polymer Chemistry, Waseda University, \\ .Ohkubo, Shinjuku-ku, Tokyo 160, Japan
}

(Received February 27, 1984)

\begin{abstract}
To control the release of insulin in response to the concentration of glucose, a glucose-responsive polymer membrane was designed by combining a glucose oxidase (GOD) immobilized membrane, a sensor for glucose, with a poly(amine) membrane which regulates the permeation rate of insulin. The permeability of insulin was increased by the addition of glucose. Gluconic acid produced by an enzymatic reaction between GOD and glucose induced a decrease in the $\mathrm{pH}$ value of the medium. This caused the protonation of tertiary amino groups in the membrane resulting in an increase in the water content of the poly(amine) membrane. The permeability of insulin through a complex membrane thus increases with glucose concentration.

KEY WORDS Glucose / Insulin / Glucose Oxidase /. Poly(amine) / Membrane / Permeation / Swelling /
\end{abstract}

Recently, many investigations have been carried out on the controlled release systems of drugs utilizing polymeric materials and maintaining the concentration of drugs in plasma within a therapeutic range for extended periods of time. ${ }^{1}$ Langer et al. demonstrated that ethylene-vinyl acetate copolymers impregnated with insulin maintained diabetic blood glucose levels near normal levels for one month. ${ }^{2}$ In comparison with insulin infusion pumps, the advantage of these implantable polymer pellets is that large amounts of insulin can be concentrated in a small volume as opposed to a mechanized pumping which must be refilled daily. ${ }^{3}$ However, the insulin release rate from these pellets dose not depend on variation in blood glucose levels and minor surgery is required for their implant and retrieval.

For an ideal insulin delivery system, insulin release should be controlled directly by the amount of blood glucose present at any particular time. This requires a continual feedback between the blood glucose level and insulin release rate.

Brownlee et al. proposed a self regulating insulin delivery system based on a combination of biological modulations and controlled release. ${ }^{4}$ The design of this delivery system utilizes the concept of competitive and complementary binding behavior of concanavalin A with glucose and glycosilated insulin.

We reported in a previous paper that the permeation rate of insulin through an amphiphilic polymer membrane can be controlled by the water fraction of the membrane. ${ }^{5} \mathrm{We}$ also reported a preparation method for a polymer membrane capable of regulating the permeation of insulin in response to change in the concentration of glucose. ${ }^{6}$ The membrane

\footnotetext{
* Present address: Sagami Chemical Research Center, Nishi-ohnuma, Sagamihara, Kanagawa 229, Japan.
} 
consists of a GOD immobilized membrane which functions as a sensor for glucose and forms hydrogen peroxide by an enzymatic reaction, and a redox polymer having a nicotinamide moiety to regulate the permeation rate of insulin by an oxidation reaction with the hydrogen peroxide.

It is well known that a polymer having tertiary amino groups is protonated by a decrease in the $\mathrm{pH}$ value of a medium and the hydrophilicity of the polymer increases. ${ }^{7}$ Therefore, a glucose-responsive polymer membrane can be obtained by combining a poly(amine) membrane and GOD immobilized membrane since the $\mathrm{pH}$ value of the medium will decrease by the formation of gluconic acid produced from the reaction of GOD with glucose.

This article discusses the permeation control of insulin using a complex membrane of immobilized GOD and poly(amine) in response to the glucose concentration.

\section{EXPERIMENTAL}

\section{Materials}

$N, N$-Diethylaminoethyl methacrylate (DEA) and 2-hydroxypropyl methacrylate (HPMA) were distilled under reduced pressure in a nitrogen atmosphere and the fractions of bp $68^{\circ} \mathrm{C} / 4 \mathrm{mmHg}$ and bp $77^{\circ} \mathrm{C} / 3.5 \mathrm{mmHg}$ were used, respectively. 2,2'-Azobisisobutyronitrile (AIBN), N,N-dimethylformamide (DMF) were purified by conventional methods. Acrylamide (AAm) was purified by recrystallization from benzene. $N, N^{\prime}$-Methylenebisacrylamide (BIS), $N, N, N^{\prime}, N^{\prime}$-tetramethylethylenediamine (TMEA), ammonium persulfate (APS) were an extra pure grade and used without further purification. GOD was purchased from Sigma Co., Ltd.

Copolymerization of DEA with HPMA and Preparation of the DEA-HPMA Copolymer Membrane

In $101.2 \mathrm{ml}$ of DMF, $10.9 \mathrm{ml}$ of DEA,

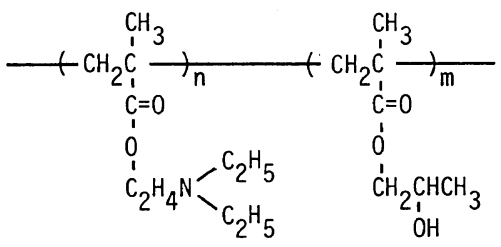

Figure 1, Structure of the DEA-HPMA copolymer.

$68.0 \mathrm{ml}$ of HPMA, and $0.148 \mathrm{~g}$ of AIBN were dissolved and the solution was placed in a polymerization tube. The tube was degassed and sealed by the conventional method. It was shaken for $24 \mathrm{~min}$ at $60^{\circ} \mathrm{C}$. The reaction mixture was then cooled and poured into an excess of acetone-hexane mixture $(1: 5)$. The precipitate was dissolved in DMF and the solution was poured again into an excess of acetone-hexane mixture. The precipitated copolymer was filtered and dried in vacuo. The mole fraction of DEA in the copolymer was 0.12 , as determined by elemental analysis. The structure of the copolymer is shown in Figure 1.

The DEA-HPMA copolymer obtained was dissolved in DMF and the solution was cast on a Teflon plate. The solvent was slowly evaporated in an oven for 3 days at $40^{\circ} \mathrm{C}$ to prepare a DEA-HPMA copolymer membrane and it was dried in vacuo. The thickness of the DEAHPMA copolymer membrane was $0.10 \mathrm{~mm}$.

\section{Preparation of the GOD Immobilized Mem- brane}

A solution of $1.31 \mathrm{~g}$ of AAm, $65.3 \mathrm{mg}$ of BIS and $10 \mathrm{mg}$ of GOD dissolved in $7 \mathrm{ml}$ of water was poured into a glass dish. To this was added $0.09 \mathrm{mg}$ of TMEA and $0.5 \mathrm{ml}$ of aqueous solution containing $25 \mathrm{mg}$ of APS. The polymerization was initiated immediately and the GOD immobilized membrane, in a transparent, gel state, was obtained. The thickness of the membrane was about $1.0 \mathrm{~mm}$. The membrane was washed with water to remove the unreacted substances and applied in the following experiment. The degree of immobilization exceeded $90 \%{ }^{6}{ }^{6}$ 
Measurement of the Water Content of the DEA-HPMA Copolymer Membrane

The DEA-HPMA copolymer membrane was immersed in a $0.1 \mathrm{M}$ sodium barbiturate buffer solution of various $\mathrm{pH}$ and swollen in a vessel thermostated at $30^{\circ} \mathrm{C}$. The swollen membrane was taken out at regular intervals and the excess solution was removed with a filter paper. The membrane was then weighed. The water content of the membrane was calculated by the following equation,

Water content $(\%)=\frac{(\mathrm{Wt} \text { of swollen membrane })-(\mathrm{Wt} \text { of dry membrane })}{(\mathrm{Wt} \text { of swollen membrane })} \times 100$

Measurement of the Amount of Insulin Permeated through the DEA-HPMA Copolymer Membrane

The following permeation experiment was carried out. The DEA-HPMA copolymer membrane was swollen in a buffer solution of given $\mathrm{pH}$ at room temperature. After the water content of the copolymer membrane reached equilibrium, the copolymer membrane was interposed between two glass cells. The permeation area of the cell was $7.07 \mathrm{~cm}^{2}$. A buffer solution $50 \mathrm{ml}$, containing $8.35 \mathrm{mg}$ of insulin was placed in one cell and $50 \mathrm{ml}$ of the buffer solution in the other. Both solutions were stirred slowly. Aliquots of the buffer solution were taken out after given periods of time and the absorbance at $274 \mathrm{~nm}$ based on insulin in the solution was measured to determine the amount of insulin which had permeated the membrane. The permeation coefficient was calculated from the slope of the permeation profile of insulin through the membrane..$^{5,6}$
Measurement of the Amount of Insulin Permeated through a Complex Membrane

The insulin permeation experment using a complex membrane was carried out as follows. The GOD immobilized membrane and preswollen DEA-HPMA copolymer membrane were combined and interposed between two glass cells. The apparatus is shown in Figure 2. In the insulin permeation experiment without glucose, $50 \mathrm{ml}$ of $1 \mathrm{mM}$ sodium phosphate buffer solution ( $\mathrm{pH} 7.10)$ containing $0.95 \mathrm{~g}$ of $\mathrm{Na}_{2} \mathrm{SO}_{4}$ and $8.35 \mathrm{mg}$ of insulin were placed in the cell facing the DEA-HPMA copolymer membrane and $50 \mathrm{ml}$ of the buffer solution were added to the cell facing the GOD immobilized membrane. In the experiment with glucose, $50 \mathrm{ml}$ of the buffer solution containing a given amount of glucose were placed in the GOD immobilized membrane side. The amount of permeated insulin was then determined spectrophotometrically with a Shimadzu UV-240 Spectrophotometer

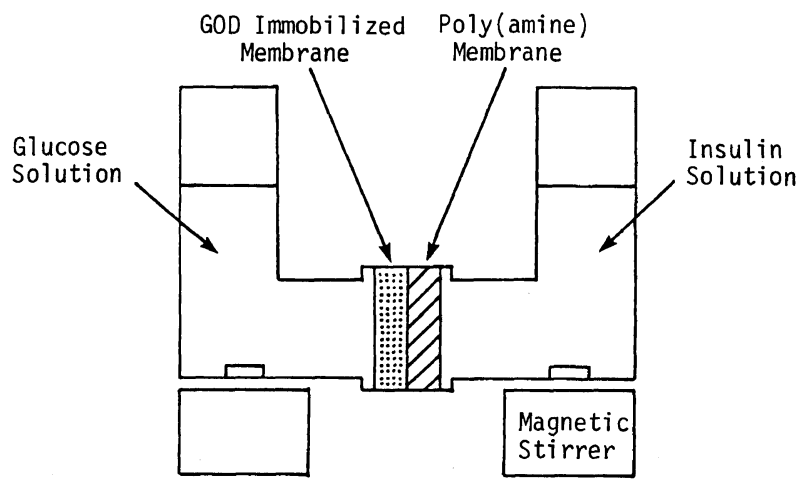

Figure 2. Apparatus for the insulin permeation experiments. 
as mentioned above.

\section{RESULTS AND DISCUSSION}

Swelling Behavior of the DEA-HPMA Copolymer Membrane

Figure 3 shows the $\mathrm{pH}$ dependence of the equilibrium water content of the DEA-HPMA copolymer membrane. The water content increased with decreasing the $\mathrm{pH}$ of the medium, there was a particularly large increase in the water content below $\mathrm{pH} 6.80$. It was confirmed that the water content of the poly(HPMA) membrane was independent of the $\mathrm{pH}$ of the medium and thus the change in water content of the DEA-HPMA copolymer membrane resulted from the introduction of the DEA moiety. The DEA moiety was considered to be protonated by 'a decrease in the $\mathrm{pH}$ of the medium followed by an increase in the hydrophilicity of the DEA moiety. This explains the $\mathrm{pH}$ dependence of the water content.

To investigate the reversibility of the change in the water content of the DEA-HPMA copolymer membrane induced by $\mathrm{pH}$ change, its time dependence was measured, when the $\mathrm{pH}$ of the medium was changed reversibly. The results are shown in Figure 4. When the preswollen membrane in a medium of a low $\mathrm{pH}$ ( $\mathrm{pH} 6.50$ or 6.65) was put in one of high $\mathrm{pH}$ (pH 7.40), the water content decreased. With a subsequent decrease in $\mathrm{pH}$, the water content recovered its original value. Thus, the water content of the DEA-HPMA copolymer membrane changes reversibly by change in the $\mathrm{pH}$ of the medium.

\section{Permeation of Insulin through the DEA-} HPMA Copolymer Membrane

Figure 5 shows the relation between the permeation coefficient of insulin through the DEA-HPMA copolymer membrane and the $\mathrm{pH}$ of the medium. The permeation coefficient increased with decreasing $\mathrm{pH}$, and there was a particularly large increase in the permeation coefficient below pH 6.80. This tendency was

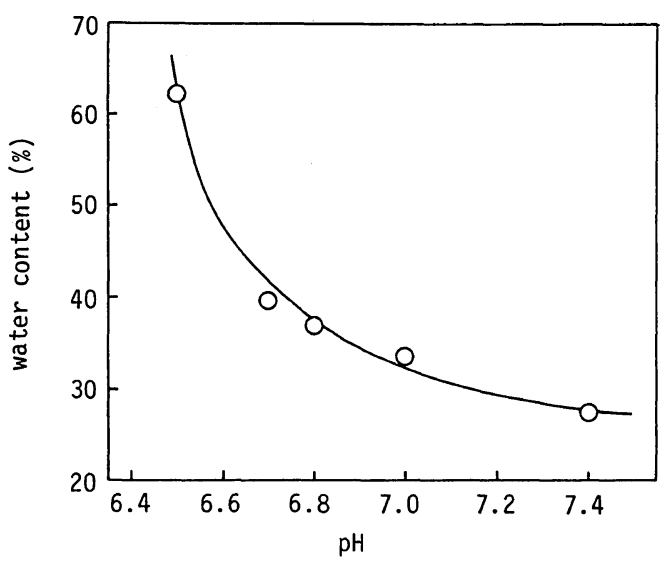

Figure 3. $\mathrm{pH}$ dependence of water content of the DEA-HPMA copolymer membrane at $30^{\circ} \mathrm{C}$.

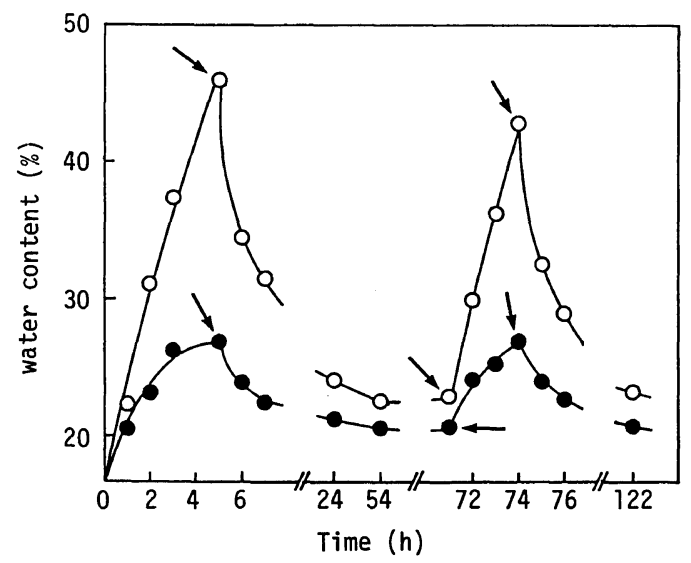

Figure 4. Reversible change in water content of the DEA-HPMA copolymer membrane induced by the $\mathrm{pH}$ change at $30^{\circ} \mathrm{C}$ : (A) $\mathrm{pH} 6.50$; (B) $\mathrm{pH} 7.40$; (C) $\mathrm{pH} 6.65$.

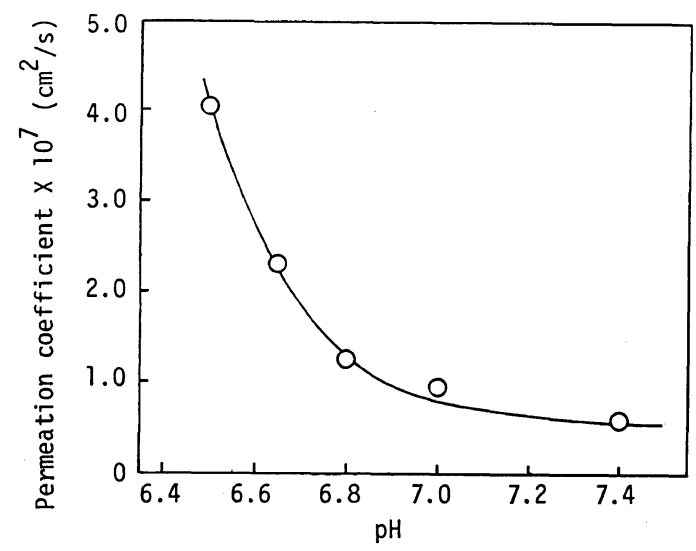

Figure 5. $\mathrm{pH}$ dependence of the permeation coefficient of insulin through the DEA-HPMA copolymer membrane at $30^{\circ} \mathrm{C}$. 


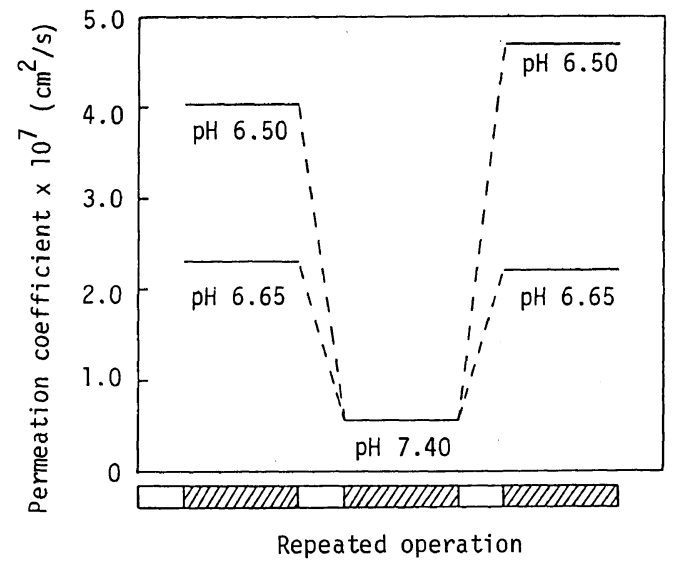

Figure 6. Various permeation coefficients of insulin through the DEA-HPMA copolymer membrane: $(\square)$ soaking, ( $)$ permeation.

also observed for the water content of the membrane (see Figure 3). It is generally known that the permeability of a substance through a swollen polymer membrane depends on its water content. ${ }^{8}$ In such a system, it is considered that an increase in the permeation coefficient at a low $\mathrm{pH}$ value is induced by an increase in the water content of the DEAHPMA copolymer membrane.

Figure 6 shows the results of permeation experiments of insulin through the DEAHPMA copolymer membrane with continuous change in the $\mathrm{pH}$ of the medium. It is clear that the permeation coefficient of insulin through the membrane changes reversibly with $\mathrm{pH}$. change. This corresponds to the swelling behavior of the DEA-HPMA copolymer membrane shown in Figure 5.

Thus, if the $\mathrm{pH}$ is changed through an appropriate chemical reaction, the permeability of insulin through the DEA-HPMA copolymer membrane should also change in response to the reaction.

\section{Permeation of Insulin through a Complex Membrane Composed of the GOD Immo- bilized and DEA-HPMA Copolymer Mem- branes}

The change in the water content of the

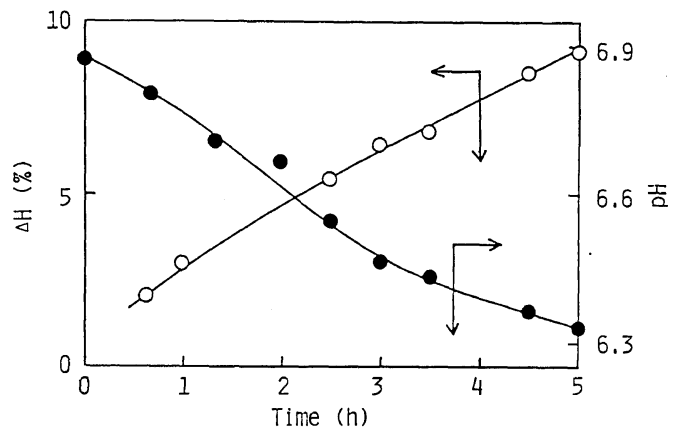

Figure 7. Changes in water content of the DEAHPMA copolymer membrane and $\mathrm{pH}$ of the medium in $1 \mathrm{mM}$ phosphate buffer solution containing $0.1 \mathrm{M}$ glucose with the GOD immobilized membrane at $30^{\circ} \mathrm{C}: \Delta H$ represents the change in membrane water content.

DEA-HPMA copolymer membrane induced by an enzymatic reaction GOD and glucose was investigated. The experiment was carried out in $1 \mathrm{mM}$ phosphate buffer solution containing $1 \mathrm{mM}$ of glucose. The time dependence of change in water content and $\mathrm{pH}$ of the medium is shown in Figure 7. The $\mathrm{pH}$ of the medium decreased and the water content of the DEA-HPMA copolymer membrane increased at the same time with an increase in the reaction time. A decrease in $\mathrm{pH}$ was considered to result from the enzymatic reaction between GOD and glucose to form gluconic acid. The increase in water content of the DEA-HPMA copolymer membrane was attributed to protonation of the tertiary amino groups of the copolymer in response to a decrease in the $\mathrm{pH}$.

Therefore, since the water content of the DEA-HPMA copolymer membrane can be changed by the enzymatic reaction between GOD and glucose, the permeation of insulin through the polymer membrane may change in response to the glucose concentration. A schematic representation of the glucose-induced permeation control of insulin using a complex membrane composed of the GOD immobilized membrane and a polyelectrolyte membrane having tertiary amino groups such as the DEA-HPMA copolymer membrane is shown 
in Figure 8.

Figure 9 shows the results of insulin permeation through the complex membrane at various concentrations of glucose. It can be seen that the permeation rate of insulin in the presence of glucose was greater than that without glucose. The permeability of insulin through the GOD immobilized membrane alone was not changed by the addition of glucose and was about 10 times greater than that through the DEA-HPMA copolymer membrane. ${ }^{6}$. Moreover, the permeation rate of insulin through the complex membrane composed of the crosslinked poly(AAm) membrane instead of the GOD immobilized membrane, and the DEA-HPMA copolymer membrane in the medium with $0.1 \mathrm{M}$ glucose was

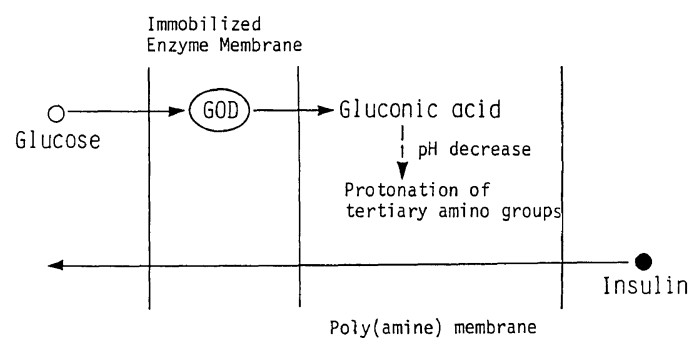

Figure 8. Schematic representation ${ }^{*}$ of the glucoseresponsive polymer membrane consisting of a poly(amine) and GOD immobilized membranes. almost the same as that without glucose. In this case, the enzymatic reaction between GOD and glucose did not occur. Therefore, this increase in the permeation rate was attributable to the enzymatic reaction between GOD and glucose. In our previous article, the permeability of insulin through an amphiphilic polymer membrane was found to be influenced by the water content of the membrane..$^{5}$ Thus, increase in the permeation rate of insulin through the complex membrane was considered due to the change in the water content of the DEA-HPMA copolymer membrane induced by the enzymatic reaction between GOD and glucose.

In the initial period of the permeation profile, the permeation rate of insulin in the medium with $0.2 \mathrm{M}$ glucose was greater than that with $0.1 \mathrm{M}$ glucose, but later both rates became nearly equal. The difference in the initial permeation rates was possibly due to the different initial rates of the enzymatic reaction owing to the different glucose concentration.

Thus, it may be concluded that the permeation control of insulin in response to glucose concentration is possible by using a complex membrane consisting of the GOD immobilized and DEA-HPMA copolymer membranes. Since the change in the permeation of

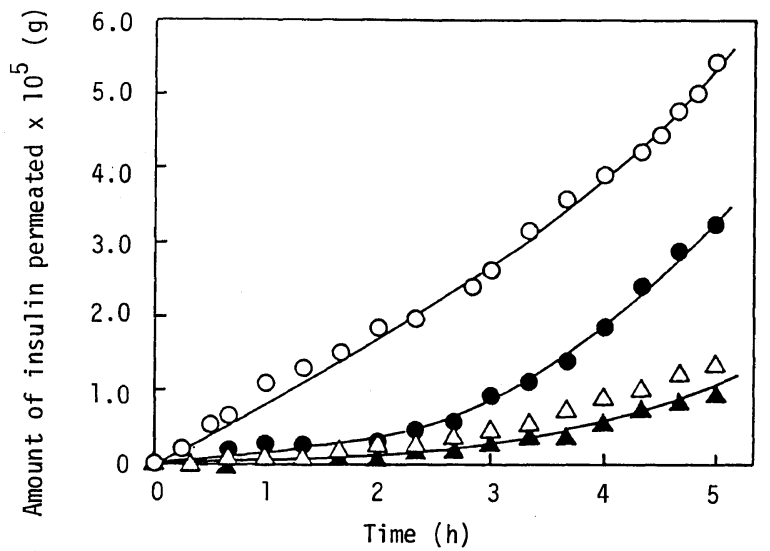

Figure 9. Permeation profile of insulin through a complex membrane consisting of the GOD immobilized and DEA-HPMA copolymer membranes at $30^{\circ} \mathrm{C}$. Glucose concentration: (O) $0.2 \mathrm{M} ;(\boldsymbol{O}) 0.1 \mathrm{M} ;(\boldsymbol{\Delta}) 0 \mathrm{M} ;(\triangle)$ $0.1 \mathrm{M}$ using the crosslinked poly(AAm) membrane in stead of the GOD immobilized membrane. 
insulin through the DEA-HPMA copolymer membrane is reversible in response to the $\mathrm{pH}$ of the medium, the reversible permeation control of insulin is possible through the change in the glucose concentration by the complex membrane.

Glucose-responsive polymer membranes such as this complex membrane should be applicable to the controlled release of insulin as a responsive function of glucose concentration.

Acknowledgement. We thank Dr. Teruo Okano and Dr. Kazunori Kataoka, Tokyo Women's Medical College, for their valuable comments made in regard to this research.

\section{REFERENCES}

1. A. C. Tanquary and R. E. Lacey, Ed., "Controlled Release of Biologically Active Agents," Plenum Press, New York, N.Y., 1974.

2. R. S. Langer and N. A. Peppas, Biomaterials, 2, 201 (1981).

3. (a) P. Bassman and R. D. Schults, Horm. Metab. Res., 4, 413 (1972); (b) A. C. Mauer, American Scientist, 67, 422 (1979).

4. M. Brownlee and A. Cerami, Science, 206, 1190 (1979).

5. K. Ishihara, M. Kobayashi, and I. Shinohara, Polym. J., 16, 647 (1984).

6. K. Ishihara, M. Kobayashi, and I. Shinohara, Makromol. Chem. Rapid Commun., 4, 327 (1983).

7. F. Alhaique, M. Marchetti, F. M. Riccieri, and E. Santucci, J. Pharmacol., 33, 413 (1981).

8. H. Yasuda, C. E. Lamaze, and L. D. Ikenberry, Makromol. Chem., 118, 19 (1968). 